\title{
Editorial
}

\section{Deflection Of Steel-Core Sandwich Panels In Weak Direction}

\section{Shao Yongbo*}

\author{
School of Civil Engineering, Yantai University, Yantai, PR China
}

The aim of this special issue is to provide a simplified method for estimating the deflection of sandwich panels with steel cores. Sandwich panels have potential advantages especially in resisting dynamic or blast action because they can absorb much more energy before fracture failure. In addition, low weight, high relative stiffness, and fast construction of sandwich panels are attractive for consideration in designing ships, buildings and bridges etc.

A typical sandwich panel consists of two facing sheets and a series of cores. Hot rolled or cold formed steels have both high material strength and relative high flexural and torsion stiffness, and thus they are selected as the cores in sandwich panels to provide higher capacity and serviceability. A key problem to produce a steel-core sandwich panel is fabrication and reliability of the connection. Fortunately, this problem is overcome when laser welding technique is used. This technique can provide quite greater welding speed compared to conventional arc welding with electrode. Moreover, a relatively easier control for weld quality can be given, and welding residual stresses can be reduced effectively.

In sandwich panels, steel cores are placed in same direction. In this placing direction, a steel-core sandwich panel has much stronger flexural strength, and this direction is then called strong direction. However, in the direction perpendicular to the strong direction (so-called weak direction), the sandwich panel has weak shear stiffness because the steel cores are not continuous in this direction. In the strong direction, the sandwich panel behaves just like a classical plate. For brevity, the sandwich panel can be simplified into an Ibeam (by changing some material constants), in which two facing plates are assumed to be flanges and steel core is simplified as web. Since the steel cores are placed with a certain space in the weak direction, the sandwich panel can be thought to have no web between any adjacent steel cores.

*Address correspondence to this author at the School of Civil Engineering, Yantai University, Yantai, PR China; Tel: +86-535-6905138;

Fax:+86-6902606; E-mail: cybshao@ytu.edu.cn
Therefore, the sandwich panel has low capacity in resisting shear deformation in the weak direction.

In the literature, many researchers paid much attention in this field. They studied the behavior of steel-core sandwich panels by using theoretical derivation, numerical simulation or experimental measurements. Although different models have been presented based on the above studies, it is still essential to provide simple yet reliable method for design purposes. Steel-core sandwich panel is a composite structure, and it has different mechanical properties in two directions. In theory, both plate/shell mechanics and mechanics of composite materials should be used to analyze this structure. This will bring much complicated solving process and expression of final equations. Due to this reason, a try to obtain simple yet accurate semi-empirical equation for predicting the behavior of steel-core sandwich panel becomes the objective of this special issue.

This special issue includes overall 6 papers. The topics of these papers focus on the prediction of the deflection of steel-core sandwich panels in weak direction. Six types of steel-cores, namely web-core, box-core, truss-core, C-core, V-core and Z-core, are selected in the sandwich panels. Based on some reasonable assumptions, the authors used fundamentals of structural mechanics to analyze the deflection of each type of steel-core sandwich panel. They provide relatively simple equations for calculating such deflection. For design purposes, the validity range of these equations is also given based on results of corresponding parametric study. It should be mentioned that all the proposed equations are deduced from some simplifications and assumptions. These simplifications and assumptions can be valid only when the geometry of a steel-core sandwich panel lies in a limited range. This means a validity range is necessarily considered when these equations are used to calculate the deflections.

Finally, all these papers in the special issue have been reviewed by some famous experts, and they are also revised according to the reviewers' comments and suggestions. I hereby express my sincere thanks to the reviewers for their valuable work. Also, I wish this issue can provide some useful reference for researchers in this field.

(C) Shao Yongbo; Licensee Bentham Open.

This is an open access article licensed under the terms of the Creative Commons Attribution Non-Commercial License (http://creativecommons.org/licenses/by-nc/3.0/) which permits unrestricted, non-commercial use, distribution and reproduction in any medium, provided the work is properly cited. 